\section{Journal of what?}

\author{
William H. Press
}

Journal of Scientific Computing. Editor Steven A. Orszag. Plenum. 4/yr. US \$135, elsewhere \$155.

LET US recall the old tale of 'nail soup', wherein an enterprising stranger astounds the rustic villagers by making a delicious soup from only boiling water and a nail plus, of course, the various ingredients that they trade him for a chance to taste the miraculous pottage. The launch of a new scientific journal is something like that: publisher and editor seed the first issue with a few invited papers - not necessarily of the quality or breadth that they hope to attract - and then wait expectantly for an influx of tasty contributions.

Like the enterprising stranger, a founding editor tries to promise a lot, sometimes more than he can deliver: the Journal of Scientific Computing, wrote the editor, Steven A. Orszag, in its first issue, "is the forum for state-of-the-art developments in scientific computing and its applications" (italics his). That has simply not transpired. What we have instead is a modest, highly specialized journal that is firmly centred on Orszag's research interests, which I would take to be (i) computational fluid dynamics, (ii) especially incompressible flows, (iii) especially spectral (for example Chebyschev) methods. This is an interesting and active area; but by no stretch of the imagination can it be said to be the unique centre of 'scientific computing', whatever that is.

Some of the more interesting papers published, at least by the end of 1987, are variations that range more widely from the theme, for example those on the implementation of some techniques on parallel computers and on efficient methods for some eigenvalue problems. The occasional paper on something as different from fluids as multimode waveguide analysis seems, however, to be the exception and not the rule.

Indeed, what is the core of scientific computing? Is it computational fluid dynamics? Is it finite difference approximation to partial differential equations? Is it image deconvolution, or three-dimensional visualization? Is it quantum chemistry, or geophysical fluid dynamics, or

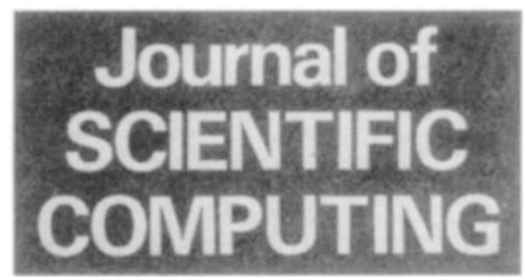

lattice gauge theory, or Monte Carlo, or the collection of things represented in the well-established Journal of Computational Physics? I suspect that as regards defining scientific computing as a field in 1988, we are not yet beyond Justice Potter Stewart's definition of pornography in 1964 (Jacobellis v. Ohio): “. . . I know it when I see it".

The world does need a journal of scientific computing, but a broader consensus on what that field includes is surely a prerequisite. For now, specialized journals - even when as grandly named as this one-remain the rule.

William H. Press is Professor of Astronomy and Physics in the Harvard-Smithsonian Center for Astrophysics, 60 Garden Street, Cambridge, Massachusetts 02138, USA

\section{Tools of the trade}

Edwin Metcalfe \& Stephen J. Haswell

Journal of Chemometrics. Editor-in-chief Bruce R. Kowalski. Wiley. 4/yr plus supplement. $£ 125, \$ 225$.

Chemometrics and Intelligent Laboratory Systems. Editor-in-chief D.L. Massart. Elsevier. 8/yr. Dfl 564, \$275.

Chemometrics is usually defined as the application of statistical, computational and mathematical methods to analytical chemistry, which in its broadest sense includes applications to areas as diverse as geochemistry and microbiology. The subject has undergone a period of tremendous growth and maturation over the past few years, so it is no accident that two journals devoted to it should appear recently and almost simultaneously.

Both Journal of Chemometrics and
Chemometrics and Intelligent Laboratory Systems provide wide coverage of the area through the publication of original papers and general information. Most of the articles in both journals are concerned with multivariate data analysis and reduction methods such as principal component, partial least squares and cluster analysis. Much of this work is aimed at extracting the essential information from multi-dimensional data sets for the purposes of classification, calibration or prediction. The alternative approaches of experimental design and optimization, in which the object is to reduce the experimentation required to yield the necessary information, are represented, although to a lesser extent. Other areas covered include the assessment and applications of expert systems and artificial intelligence, and signal-processing methods such as Kalman filtering and correlation chromatography.

The editorial approaches differ, in that
Journal of Chemometrics is more theoretically orientated; it is more formal in its presentation, and although the papers identify analytical problems their emphasis is more on the underlying theory. Chemometrics and Intelligent Laboratory Systems, on the other hand, contains a roughly equal mix of theoretical and applied papers; here the stress is upon the more practical aspects, as emphasized by the excellent tutorial section. This section addresses specific chemometric techniques, such as optimization of HPLC separation or soft modelling, and describes their use in a readable and comprehensible way. Both journals have book and software reviews and a diary section, but these are better presented and far more detailed in Chemometrics and Intelligent Laboratory Systems, which also includes a useful and sometimes provocative editorial slot, and meeting reports. In this way the journal appears to operate as an information forum for members of the Chemometrics Society, which acts as its sponsor.

Many of the original papers in both journals are of a high standard and could, with stylistic changes, be published in either one of them. Both have a reasonable rate of production, most articles and short communications being published within a year of receipt, and the illustrations and format are satisfactory.

These two journals are welcome in providing a forum for practising chemometricians, but it is both inevitable and necessary for the continued growth of the subject that many papers, particularly those which produce a faster or more reliable interpretation of real data, will continue to be published in other journals, to maintain and generate further dialogue with non-chemometricians. If chemometrics can be seen as essentially a tool kit, Journal of Chemometrics and Chemometrics and Intelligent Laboratory Systems are for reports of investigations into the advantages, developments and limitations of the various tools, while papers reporting the use of the tools as aids to wider investigations will continue to appear in analytical and more general journals.

Both of the journals reviewed here are reasonably priced and are essential reading for the practising chemometrician. The part-time chemometrician who is interested primarily in practical applications should, ideally, also consult both of them. But, if forced to choose, we would plump for Chemometrics and Intelligent Laboratory Systems, not just for its more applied nature but for the tutorials provided.

Edwin Metcalfe and Stephen J. Haswell are in the School of Chemistry, Thames Polytechnic, Wellington Sireet, Woolwich, London SE186PF, UK. Stephen Haswell is Secretary of the Royal Society of Chemistry Analytical Division's Chemometrics Group. 